\title{
A word from OLAW and APHIS
}

In response to the questions posed in this scenario, the Office of Laboratory Animal Welfare (OLAW) and the Animal and Plant Health Inspection Service (APHIS) offer the following clarification and guidance, with two caveats: (i) the OLAW response presumes that the studies are funded by the Public Health Service; (ii) the APHIS response would apply if the species in question was a USDA-regulated species; at present, the species in the scenario (Mus musculus bred for research) is not.

This column presents the reader with the following questions: Is the transportation and euthanasia of the mice a protocol violation?

If so, is it reportable to NIH/OLAW, and which IACUC should make the report? How can future problems of this nature be prevented?

In the scenario, two activities, transportation of the animals and change in location of the euthanasia procedure, were not approved by the IACUC with jurisdiction over the mice, the university's IACUC. The biotechnology company's IACUC acted outside of its oversight authority; therefore, the actions taken by its employees constitute a noncompliance. This is reportable to OLAW as a significant change implemented without IACUC approval ${ }^{1}$. In this particular circumstance, assuming the biotechnology company is the primary grantee, it bears the responsibility for compliance. Its IACUC would be expected to report the incident through the Institutional Official and to develop and implement corrective actions to prevent a repeat occurrence. OLAW will accept noncompliance reports from either the primary grantee or the performance site, depending on the nature of the noncompliance and which site is best able to take corrective action.

There is no federal requirement for dual review and approval of research activities. Therefore, review by both IACUCs is not indicated ${ }^{2}$. However, having a written agreement that clearly defines oversight of transportation between facilities and IACUC review of amendments that affect both institutions would provide a satisfactory resolution and preventive strategy. Such an agreement would meet the recommendations in the Guide for the Care and Use of Laboratory Animals and the requirements of the NIH Grants Policy Statement on written agreements 3,4 . At a minimum, the agreement must also address ways in which the Public Health Service Policy on Humane Care and Use of Laboratory Animals requirement for review and approval of proposed animal activities and semiannual facilities review by an IACUC will be met ${ }^{5}$.

For a regulated species, section 2.31(e) (3) of the Animal Welfare Act Regulations (AWARs) requires a complete description of the proposed use of an animal to be written in the animal protocol ${ }^{6}$. This includes transportation to another facility for euthanasia and tissue harvest. An amendment can be added to the Great Eastern protocol to indicate that euthanasia will be performed at New Antigen, and transportation can begin after the IACUC has approved the amendment. Conditions during transportation must be in accordance with AWAR requirements for the species being used. Record-keeping requirements must be in accordance with AWAR section 2.35 where applicable ${ }^{6}$.

1. Office of Laboratory Animal Welfare. Guidance on Prompt Reporting to OLAW under the PHS Policy on Humane Care and Use of Laboratory Animals. Notice NOTOD-05-034. (National Institutes of Health, Washington, DC, 24 February 2005, updated 21 February 2013). <http://grants.nih.gov/grants/guide/notice-files/ not-od-05-034.html>

2. Public Health Service. Policy on Humane Care and Use of Laboratory Animals_Frequently Asked Questions. Protocol Review, Question No. D.8. (US Department of Health and Human Services, Washington, DC, 2006, revised 2013). <http://grants.nih.gov/grants/olaw/faqs.htm\#d8>

3. Institute for Laboratory Animal Research. Guide for the Care and Use of Laboratory Animals 8th edn. 15 (National Academies Press, Washington, DC, 2011).

4. US National Institutes of Health. NIH Grants Policy Statement; Part II Terms and Conditions of NIH Grant Awards, Subpart B: Terms and Conditions for Specific Types of Grants, Grantees, and Activities, 15.2.1 Written Agreement. (US National Institutes of Health, Bethesda, MD, 2012). <http://grants.nih.gov/grants/ policy/nihgps_2012/nihgps_ch15.htm\#>

5. Public Health Service. Policy on Humane Care and Use of Laboratory Animals (US Department of Health and Human Services, Washington, DC, 1986; amended 2002).

6. Code of Federal Regulations, Title 9, Ch. 1, Part 2, Subpart C, §2.31(e)(3) and §2.35.

\section{Patricia Brown, VMD, MS, DACLAM}

Director

OLAW, OER, OD, NIH, HHS
Chester Gipson, DVM

Deputy Administrator

USDA, APHIS, AC agreements are essentially formal written communications. In this case, sufficient lines of communication were not in place between Great Eastern and New Antigen, resulting in confusion.

In our opinion, this violation is minor because New Antigen and Great Eastern had approved protocols. Although the situation was not universally communicated, it was approved. The removal of animals without permission is concerning, but none of the procedures conducted caused undue harm to the animals or occurred without IACUC oversight. This scenario does not address whether New Antigen's veterinary staff approved the import of animals or whether there was an existing relationship between New Antigen and Great Eastern that negated the need for health histories or quarantine regarding the movement of animals.

Great Eastern's IACUC approved the entire study to be completed at the school, including the euthanasia of the animals. Since Great Eastern's IACUC had not approved movement of the animals to New Antigen, the transportation and euthanasia of the mice at New Antigen, in the eyes of Great Eastern, would be a protocol violation and would require reporting to OLAW. Since Great Eastern had taken responsibility for housing New Antigen's animals, Great Eastern should report the violation to OLAW. However, it should be reported as a 